\title{
The Influence of Search Engines on Preferential Attachment
}

\author{
Alan Frieze, Juan Vera, and Soumen Chakrabarti
}

\begin{abstract}
There is much current interest in the evolution of social networks, in particular, the World Wide Web graph, through time. "Preferential attachment" and the "copying model" are well-known models that explain the observed power-law degree distribution of the graph reasonably well. However, existing evolution models do not include the significant influence of search engines on how webpage authors find existing pages and create links to them. Recent applied work has raised the concern that highly popular search engines limit the attention of authors to a small set of "celebrity" URLs, for any query. Page authors frequently (with probability $p$ ) locate pages using a search engine. Then they link to popular pages among those they visit. We initiate an analysis of this more realistic process, show that the celebrity nodes eventually accumulate a constant fraction of all links created with high probability (whp), and show that the degrees of the other nodes still follow a power-law distribution, but with a steeper power: $\operatorname{Pr}($ degree $=k) \propto k^{-(1+2 /(1-p))}$ whp. Our analysis adds evidence to the recent concern that search engines offer new webpages a steep, self-sustaining barrier to entry to well-connected, entrenched web communities.
\end{abstract}

\section{Introduction}

The evolution of the World Wide Web graph (Web graph, for short) through time has been subject to intense modeling, measurements, and analysis in recent years. Early measurements on the graph of webpages (nodes) and hyperlinks (edges) showed that degrees of nodes were distributed according to a power law. Barabási and Albert were among the first to propose a generative model of the World Wide Web, called preferential attachment, which leads to a power-law distribution $\operatorname{Pr}($ degree $=k) \propto k^{-3}$, i.e., a power of 3 [Barabási and Albert 99].

(C) A K Peters, Ltd.

1542-7951/06 $\$ 0.50$ per page 
Introducing random links some fraction of the time allowed Pennock et al. to bring the power closer to empirically observed data (2.1 for in-degree and 2.38 for out-degree) [Pennock et al. 02].

Preferential attachment explains a power-law degree distribution but not the presence of a large number of bipartite cliques in the Web graph. Kleinberg et al. were the first to propose a copying model in which the author of a newborn page $u$ picks a random reference page $v$ from the web and, with some probability, copies out-links from $v$ to $u$ [Kleinberg et al. 99]. Kumar et al. analyzed the copying process to show that it, too, leads to a power-law degree distribution with a power of approximately 2 [Kumar et al. 00], which is closer to empirical observation. The copying model naturally explains the presence of bipartite cliques.

Both the preferential attachment and the copying model assume organic evolution of the Web graph, without any powerful central entity influencing a large number of webpage authors with regard to how they link to existing pages. This is precisely the role that search engines like Google or Yahoo! fulfill. Webpage authors learn about a topic by launching queries to a search engine. The search engine responds with a limited number (10-20) of hits per page, and users rarely foray beyond the first 1-2 pages of hits [Cho and Roy 04].

With web search proliferating to over five billion searches per month ${ }^{1}$ as of February 2006, many webpage authors presumably create links to pages that they found by using a search engine. In other words, with some probability every link in the Web graph owes its existence to a search engine, and therefore, the evolution of the Web graph has been influenced permanently and pervasively by the existence of search engines.

In their early days, search engines merely observed and exploited the Web graph (specifically, links to a page) for ranking. Now, they are unquestionably influencing the evolution of Web graph as well. Most search engines today pay attention to in-degree and PageRank [Brin and Page 98] while ranking results. This can potentially set up a "virtuous circle of limelight": a search engine ranks a page highly, authors find the page more often, some of them link to it, raising its in-degree and PageRank, which leads to a further improvement or entrenchment of its rank.

The virtuous circle can be brutal to new pages and sites: Cho and Roy estimate that the time taken for a page to reach prominence can be delayed by a factor of over 60 if a search engine diverts clicks to entrenched pages [Cho and Roy 04]. In a theoretical setting, Drinea et al. analyze a balls-and-bins process with a related feedback mechanism and show that positive feedback leads to a

\footnotetext{
${ }^{1}$ http://www.nielsen-netratings.com/pr/PR_033006_UK.pdf
} 
rapid landslide victory for the winning bin [Drinea et al. 02]. Pandey et al. confirm that introducing some randomness in the ranking function creates a better exploration-exploitation trade-off, avoiding the worst effects of the virtuous circle [Pandey et al. 05].

Having some empirical understanding of the effect of search engines on the evolution of page popularity for search applications, we are interested in directly modeling the evolution of the Web graph under the influence of a search engine.

\section{I.I. Our Model}

We wish to model how the Web graph evolves if authors use search engines to decide on links that they insert in new pages. In particular, we are interested in the degree distribution and whether and how this distribution deviates from the power-law form derived in earlier work.

For simplicity, like Barabási et al., we model the Web graph as undirected. Following Cho and Roy, we also make the simplifying assumption that the query to the search engine is fixed and the search engine, like a bestseller list, returns some fixed number of response URLs (nodes in the Web graph), ordered according to their degree at the end of the previous time step. We can also interpret such a list as a per-topic listing provided by a directory like Yahoo! or DMoz, and limit our analysis to one topic at a time, without loss of generality.

The growth process we seek to analyze generates a sequence of (multi-)graphs $G_{t}, t=1,2, \ldots$ The graph $G_{t}=\left(V_{t}, E_{t}\right)$ has $t$ vertices and $m t$ edges. The process has only two important parameters $p$ (a probability) and $N$ (the maximum number of "celebrity" nodes listed by the search engine).

We introduce some notation:

- $\operatorname{deg}_{t}(x)$ denotes the degree of vertex $x$ in $G_{t}$;

- $D_{t}(U)$ is $\sum_{x \in U} \operatorname{deg}_{t}(x)$;

- $S_{t}$ denotes the set of at most $N$ vertices with the largest degrees in $G_{t}$ (if $t<N$ we let $\left.S_{t}=V_{t}\right)$;

- $z_{t}$ is the smallest degree of vertices in $S_{t}$;

- $Z_{t}$ is the largest degree of vertices in $V_{t} \backslash S_{t}$;

- $d_{k}(t)$ denotes the number of vertices of degree $k$ at time $t$ in the set $V_{t}-S_{t}$;

- $\bar{d}_{k}(t)$ is defined as $\mathbf{E}\left[d_{k}(t)\right]$, the expectation being over the random hyperlinking choices made by nodes (described next). 

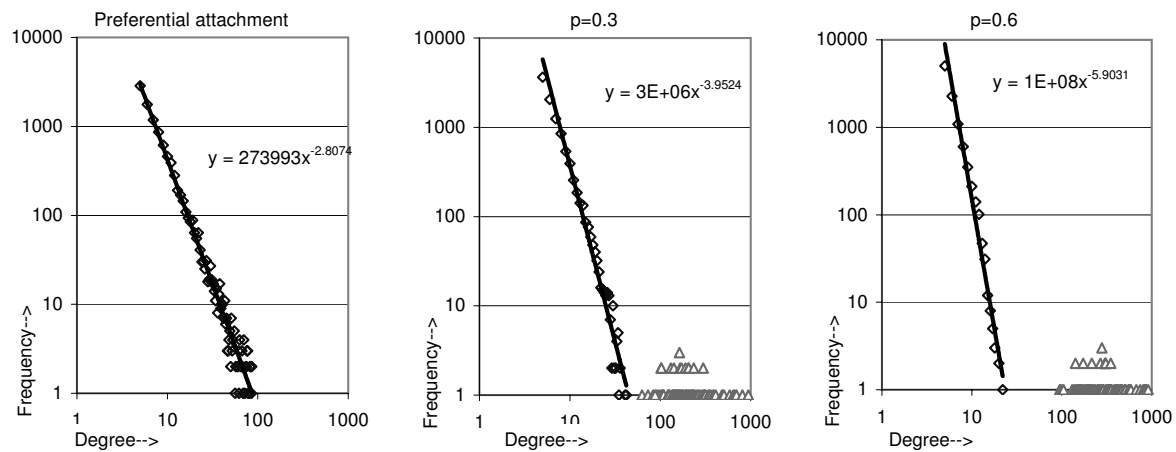

Figure I. The presence of a search engine in our model makes the power in the degree power law more negative and, with increasing $p$-(a) $p=0$, (b) $p=0.3$, (c) $p=0.6$ - separates out the celebrities from the non-celebrities $(N=100$, $|V|=10000$, and $m=5)$.

We use process $\mathcal{P}$ to generate the graph sequence $G_{t}=\left(V_{t}, E_{t}\right)$, for $t=$ $1,2, \ldots, n$ :

Definition I.I. (Process $\mathcal{P}$.)

Time step I. The process is initialized with graph $G_{1}$, which consists of an isolated vertex $x_{1}$ and $m$ loops.

Time step $\mathrm{t}>\mathrm{I}$. We add a vertex $x_{t}$ to $G_{t-1}$. We then add $m$ random edges $\left(x_{t}, y_{i}\right), i=1,2, \ldots, m$ incident with $x_{t}$, where $y_{i}$ are nodes in $G_{t-1}$. For each $i$,

(a) with probability $p$ we choose $y_{i} \in S_{t-1}$,

(b) with probability $q=1-p$, we choose $y_{i} \in V_{t-1}$.

In both cases $y_{i}$ is selected by preferential attachment within the target subset of old nodes, i.e., for $x \in U$,

$$
\operatorname{Pr}\left(y_{i}=x\right)=\frac{\operatorname{deg}_{t-1}(x)}{D_{t-1}(U)},
$$

where $U=S_{t-1}$ or $U=V_{t-1}$ as the case may be. 


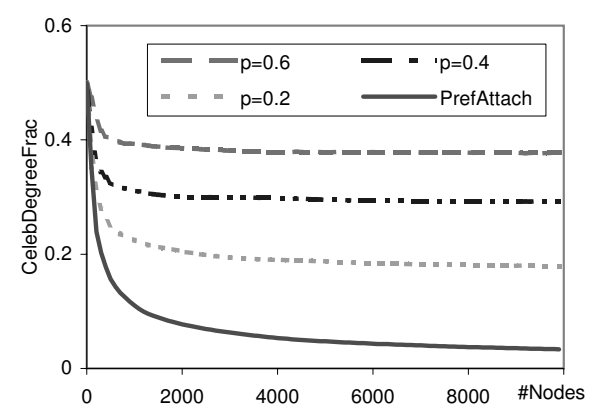

(a) $N=10, n=10000$, and $m=5$

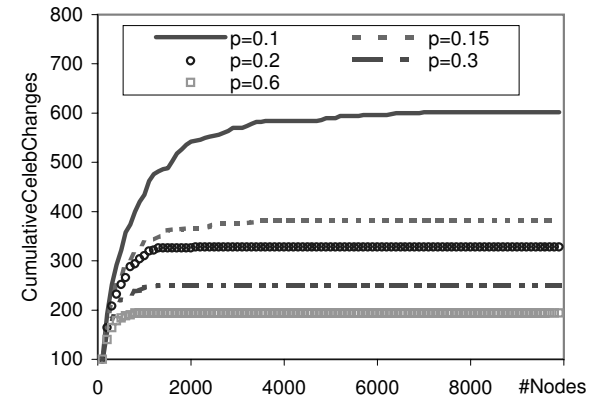

(b) $N=100, n=10000$, and $m=5$

Figure 2. (a) The total degree of the celebrities as a fraction of (twice) the number of edges added to the graph differs significantly in behavior between preferential attachment vs. our model. (b) The celebrity list becomes effectively fixed very early on in the graph evolution process, and the cumulative number of celebrity shuffles levels out faster with large $p$.

As Figure 1 shows, the simulated behavior of our proposed process is quite different from standard preferential attachment. With increasing $p$, the celebrities swing out far from the power-law straight line in log-log plots. Also, as $p$ increases, the power (negative slope) increases as well: at $p=0$ it is 2.8 , at $p=0.3$ it is 3.96 , and at $p=0.6$ it is 5.9 .

Furthermore, as Figure 2(a) shows, the total degree (as a fraction of twice the total number of edges added) over the celebrities goes to zero as $n \rightarrow \infty$ for preferential attachment, but in a simulation of our proposed model, the celebrities command a constant fraction of the total degree over all nodes, and this fraction grows with $p$. In Figure 2(b) we plot the cumulative number of nodes leaving or entering the celebrity list from each timestep to the next. We see that as $p$ increases, the celebrity list is determined more and more quickly.

\subsection{Our Results}

We will prove the following, where all asymptotic notation is with respect to $n$, the number of steps for which the process $\mathcal{P}$ is run (which is the same as the number of nodes).

Theorem I.2. Let $m \geq \max \{15,2 / q\}$ and $0<p<1$.

(a) Let $S_{n}=\left\{s_{1}, \ldots, s_{N}\right\}$ in decreasing order of degree. Then $\mathbf{E}\left[\operatorname{deg}_{n}\left(s_{i}\right)\right] \sim$ $\alpha_{i} n$ for every $i \leq N$, for some constant $\alpha_{i}>0$. That is, each celebrity commands a constant fraction of all edges ever generated in the graph. 
(b) There is an absolute constant $A_{1}$ such that, for every $k \geq m$,

$$
\begin{aligned}
\bar{d}_{k}(n) & =\frac{2 n}{2+m q} \prod_{i=m+1}^{k} \frac{i-1}{i+2 / q}+\tilde{O}\left(n^{q / 2}\right) \\
& =\frac{A_{1} n}{k^{1+2 / q}}+\tilde{O}\left(n / k^{2+2 / q}+n^{q / 2}\right) .
\end{aligned}
$$

The theorem and its proof confirms all the features that we see in the simulations: celebrities capture a large fraction of links, the celebrity list gets fixed quickly, and non-celebrities follow a power-law degree distribution with a power steeper than in preferential attachment.

The proof depends on showing that after time $t_{0}$ the first time that there is a considerable degree gap between the celebrity list and the non-celebrities, the probability of having a non-celebrity move to the celebrity list is very small. So, in practice the celebrity list becomes fixed. Once the celebrity list is fixed, our process $\mathcal{P}$ looks very similar to an analogous process $\mathcal{P}^{*}$ where in each step $S_{t}$ is replaced by $S_{t}^{*}=S^{*}=\left\{x_{1}^{*}, \ldots, x_{N}^{*}\right\}$ in decreasing order of degree (if $t<N$ take $\left.S_{t}^{*}=V_{t}\right)$. In other words, in process $\mathcal{P}^{*}$ we take the $N$ oldest vertices as $S_{t}^{*}$, instead of the $N$ largest-degree vertices.

Let $G_{t}$ be the sequence of graphs produced by process $\mathcal{P}$ and $G_{t}^{*}$ the sequence of graphs produced by process $\mathcal{P}^{*}$. In Section 2 we construct a coupling for $t=1,2, \ldots$ between the sequence of graphs $G_{t}$ and the sequence of graphs $G_{t}^{*}$.

In Section 3 we compute $\mathbf{E}\left[\operatorname{deg}_{n}(s)\right]$ for $s \in S_{n}$ and $\mathbf{E}\left[d_{k}(n)\right]$, conditioning on $t_{0}$ and $G_{t_{0}}$. In Section 4 we get bounds for the probability of having a small gap between celebrities and non-celebrities. Finally, in Section 5 we give the proof of Theorem 1.2.

\section{Coupling $G_{t}$ and $G_{t}^{*}$}

Let $z_{t}$ be the degree of the lowest degree vertex in $S_{t}$ and $Z_{t}$ the degree of the highest degree vertex in $V_{t} \backslash S_{t}$. We are going to prove that after a short time whp there is a significant gap between $z_{t}$ and $Z_{t}$ and then from this time on $S_{t}$ remains fixed. In this sense the graph $G_{t}$ is very similar to the graph $G_{t}^{*}=\left(V_{t}^{*}, E_{t}^{*}\right)$, constructed by process $\mathcal{P}^{*}$, where the top $N$ is fixed from the beginning (the top is fixed by age not by degree). We define $z_{t}^{*}$ and $Z_{t}^{*}$ in $G_{t}^{*}$ in an analogous way to $z_{t}$ and $Z_{t}$.

Lemma 2.I. We can couple $\mathcal{P}$ and $\mathcal{P}^{*}$ in such a way that for all $t>0$,

$$
D_{t}^{*}\left(S_{t}\right) \leq D\left(S_{t}\right), \quad z_{t}^{*} \leq z_{t} \quad \text { and } \quad Z_{t}^{*} \geq Z_{t} .
$$


Proof. To couple processes $\mathcal{P}$ and $\mathcal{P}^{*}$, we first define $\mathcal{P}^{\prime}$, a small modification of process $\mathcal{P}$. If after time step $t$ vertex $v \in V_{t}^{\prime} \backslash S_{t}^{\prime}$ has degree larger than the degree of $u$ (the minimum-degree vertex in $S_{t}^{\prime}$ ), then, instead of moving $v$ into $S_{t}^{\prime}$ and $u$ out of $S_{t}^{\prime}$, we change the endpoint of some of the last edges inserted incident to $v$ to make them incident to $u$ (leaving the other end fixed), in order to swap the degrees of $v$ and $u$. In this way the degree sequence in maintained, and $S_{t}^{\prime}$ remains fixed as the $N$ oldest vertices (i.e., $S_{t}^{\prime}=S_{t}^{*}$ ). The graph generated by $\mathcal{P}^{\prime}$ has a different edge structure to $G_{t}$, but it has the same degree sequence, thus

$$
D_{t}^{\prime}\left(S_{t}^{\prime}\right)=D\left(S_{t}\right), \quad z_{t}^{\prime}=z_{t}, \quad \text { and } \quad Z_{t}^{\prime}=Z_{t}
$$

Now let $\mathcal{P}^{\prime \prime}$ be the process where after each step we proceed almost the same way as in $\mathcal{P}^{\prime}$, except that in $\mathcal{P}^{\prime}$ if some $k$ endpoints are changed (from a vertex outside $S_{t}^{\prime}$ to a vertex in $S_{t}^{\prime}$ ), in $\mathcal{P}^{\prime \prime}$ we don't make these changes, but instead we move the endpoints of $k$ random edges chosen uniformly from the last inserted edges incident to $V_{t} \backslash S_{t}^{\prime \prime}$, to $k$ random positions chosen by preferential attachment in $S_{t}^{\prime \prime}=S_{t}^{\prime}=S_{t}^{*}$.

We can think of every edge inserted as two directed edges, then choosing by preferential attachment is equivalent to choosing a random edge uniformly and then choosing its destination vertex. This permits us to couple process $\mathcal{P}^{\prime}$ and $\mathcal{P}^{\prime \prime}$ in such way that

$$
D_{t}^{\prime \prime}\left(S_{t}^{\prime \prime}\right)=D^{\prime}\left(S_{t}^{\prime}\right), \quad z_{t}^{\prime \prime} \leq z_{t}^{\prime}, \quad \text { and } \quad Z_{t}^{\prime \prime}=Z_{t}^{\prime}
$$

Notice that process $\mathcal{P}^{\prime \prime}$ looks like process $\mathcal{P}^{*}$ except that the probability of applying step (a) of the process is greater than $p$ (and depends on $G_{t}$ ). So we can couple $\mathcal{P}^{*}$ and $\mathcal{P}^{\prime \prime}$ in such way that

$$
D_{t}^{*}\left(S_{t}\right) \leq D^{\prime \prime}\left(S_{t}^{\prime \prime}\right), \quad z_{t}^{*} \leq z_{t}^{\prime \prime}, \quad \text { and } \quad Z_{t}^{*} \geq Z_{t}^{\prime \prime} .
$$

Putting Equations (2.1), (2.2), and (2.3) together we get that we can couple $\mathcal{P}$ and $\mathcal{P}^{*}$ such that

$$
D_{t}^{*}\left(S_{t}\right) \leq D\left(S_{t}\right), \quad z_{t}^{*} \leq z_{t}, \quad \text { and } \quad Z_{t}^{*} \geq Z_{t}
$$

\section{Analysis of the Degree Distribution of $G_{t}^{*}$}

Given a time $t>0$, let $\mathcal{G}_{t}$ be the set of graphs with vertex set $\left\{x_{1}, \ldots, x_{t}\right\}$ and $m t$ edges. Notice that $G_{t}, G_{t}^{*} \in \mathcal{G}_{t}$. In this section we analyze the behavior of 
$G_{t}^{*}$ when process $\mathcal{P}^{*}$ is initialized at time $t$ with some graph $G \in \mathcal{G}_{t}$, i.e., when conditioning on $G_{t}^{*}=G$. In Lemma 3.1 we prove that $\overline{d_{k}^{*}}(n)$ follows a power law, while in Lemma 3.2 we prove that the expected $\operatorname{deg}_{n}^{*}\left(x_{i}^{*}\right)$ grows linearly with time for nodes $x_{i}^{*}$ with $i \leq N$.

Lemma 3.I. Fix a time $t_{0} \geq N$ and let $G \in \mathcal{G}_{t_{0}}$. Then, at a later time $n \geq t_{0}$, for all $k \geq m$

$$
\begin{aligned}
\mathbf{E}\left[d_{k}^{*}(n) \mid G_{t_{0}}^{*}=G\right] & =\frac{2 n}{2+m q} \prod_{i=m+1}^{k} \frac{i-1}{i+2 / q}+\tilde{O}\left(t_{0}+n^{q / 2}\right) \\
& =\frac{A_{1} n}{k^{1+2 / q}}+\tilde{O}\left(t_{0}+n^{q / 2}+n / k^{2+2 / q}\right) .
\end{aligned}
$$

Proof. Our approach to proving a power law is to find a recurrence for $\mathbf{E}\left[d_{k}^{*}(n) \mid G_{t_{0}}^{*}=G\right]$.

Notice that $\mathbf{E}\left[d_{m-1}^{*}(\tau) \mid G_{t_{0}}^{*}=G\right]=0$ for all $\tau>0$. Then for $\tau \geq t_{0}, k \geq m$,

$$
\begin{aligned}
\mathbf{E}\left[d_{k}^{*}(\tau+1) \mid G_{\tau}^{*}, G_{t_{0}}^{*}\right]= & d_{k}^{*}(\tau)+q m\left(\frac{(k-1) d_{k-1}^{*}(\tau)}{2 m \tau}-\frac{k d_{k}^{*}(\tau)}{2 m \tau}\right) \\
& +1_{k=m}+O\left(Z_{\tau}^{*} \tau^{-1}\right) \\
= & d_{k}^{*}(\tau)+q \frac{(k-1) d_{k-1}^{*}(\tau)-k d_{k}^{*}(\tau)}{2 \tau} \\
& +1_{k=m}+O\left(Z_{\tau}^{*} \tau^{-1}\right) .
\end{aligned}
$$

Here is an explanation of Equation (3.1): $q m$ is the expected number of edges involving non-celebrities. The expression following $q m$ is the probability that an additional edge convertes a vertex of degree $k-1$ to one of degree $k$ less the probability that it converts a vertex of degree $k$ into one of degree $k+1$. The $O\left(Z_{\tau}^{*} \tau^{-1}\right)$ term accounts for the addition of parallel edges.

Taking expectations with respect to $G_{\tau}^{*}$, we get

$$
\begin{aligned}
\mathbf{E}\left[d_{k}^{*}(\tau+1) \mid\right. & \left.G_{t_{0}}^{*}=G\right]=\mathbf{E}\left[d_{k}^{*}(\tau) \mid G_{t_{0}}^{*}=G\right] \\
& +q \frac{(k-1) \mathbf{E}\left[d_{k-1}^{*}(\tau) \mid G_{t_{0}}^{*}=G\right]-k \mathbf{E}\left[d_{k}^{*}(\tau) \mid G_{t_{0}}^{*}=G\right]}{2 \tau} \\
& +1_{k=m}+O\left(\mathbf{E}\left[Z_{\tau}^{*} \mid G_{t_{0}}^{*}=G\right] \tau^{-1}\right) .
\end{aligned}
$$

We consider the exact recurrence, $f_{m-1}=0$ and for $k \geq 0$,

$$
f_{k}=1_{k=m}+q \frac{(k-1) f_{k-1}-k f_{k}}{2}
$$


yielding

$$
f_{m}=\frac{2}{2+m q}
$$

and

$$
\begin{aligned}
f_{k} & =\frac{2}{2+m q} \prod_{i=m+1}^{k} \frac{i-1}{i+2 / q} \\
& =\frac{2}{2+m q} \exp \left(\sum_{i=m+1}^{k} \ln \left(\frac{i-1}{i+2 / q}\right)\right) \\
& =\frac{2 e^{-g(m, k)}}{2+m q}\left(\frac{m+1+2 / q}{k+2 / q}\right)^{1+2 / q} \\
& =\frac{2 e^{-g(m)}(m+1+2 / q)^{1+2 / q}}{2+m q} \frac{e^{O(1 / k)}}{k^{1+2 / q}}
\end{aligned}
$$

where

$$
\begin{aligned}
g(m, k) & =\left(\sum_{i=m+1}^{k} \frac{1+2 / q}{i+2 / q}-\int_{x=m+1}^{k} \frac{1+2 / q}{x+2 / q} d x\right)+\sum_{i=m+1}^{k} \sum_{l=2}^{\infty} \frac{1}{l}\left(\frac{1+2 / q}{i+2 / q}\right)^{l} \\
& =g_{m}+O\left(k^{-1}\right),
\end{aligned}
$$

and $g_{m}=\lim _{k \rightarrow \infty} g(m, k)$.

We finish the proof of the lemma by showing that there exists a constant $M>0$ such that

$$
\left|\mathbf{E}\left[d_{k}^{*}(\tau) \mid G_{t_{0}}^{*}=G\right]-f_{k} \tau\right| \leq M\left(t_{0}+\tau^{q / 2}(\ln \tau)^{3}\right)
$$

for all $\tau>0$.

Let

$$
\Theta_{k}(\tau)=\mathbf{E}\left[d_{k}^{*}(\tau) \mid G_{t_{0}}^{*}=G\right]-f_{k} \tau .
$$

Lemma 4.4 (proved later) implies that $\mathbf{E}\left[Z_{\tau}^{*} \mid G_{t_{0}}^{*}=G\right] \leq O\left(\tau^{q / 2}(\ln \tau)^{3}\right)$. So after taking expectations over $G_{\tau}^{*}$ in Equation (3.1) and substituting $\mathbf{E}\left[d_{k}^{*}(\tau) \mid G_{t_{0}}^{*}=G\right]=\Theta_{k}(\tau)+f_{k} \tau$, we see that for $k \geq m$ and $\tau \geq t_{0}$,

$$
\begin{aligned}
f_{k}(\tau+1)+\Theta_{k}(\tau+1)= & f_{k} \tau+\Theta_{k}(\tau) \\
& +q \frac{(k-1)\left(\Theta_{k-1}(\tau)+f_{k-1} \tau\right)-k\left(\Theta_{k}(\tau)+f_{k} \tau\right)}{2 \tau} \\
& +1_{k=m}+O\left(\tau^{q / 2-1}(\ln \tau)^{3}\right) .
\end{aligned}
$$


Using Equation (3.3) to eliminate the $f_{k}$, we obtain

$$
\Theta_{k}(\tau+1)=\left(1-\frac{q k}{2 \tau}\right) \Theta_{k}(\tau)+\frac{q(k-1)}{2 \tau} \Theta_{k-1}(\tau)+O\left(\tau^{q / 2-1}(\ln \tau)^{3}\right) .
$$

Let $L$ denote the hidden constant in $O\left(\tau^{q / 2-1}(\ln \tau)^{3}\right)$ of Equation (3.5). Our inductive hypothesis $\mathcal{H}_{\tau}$ is that $\left|\Theta_{k}(\tau)\right| \leq M\left(t_{0}+\tau^{q / 2}(\ln \tau)^{3}\right)$ for every $k \geq m$. It is trivially true for $\tau \leq t_{0}$. So assume that $\tau \geq t_{0}$. Then, from Equation (3.5),

$$
\begin{aligned}
\left|\Theta_{k}(\tau+1)\right| & \leq M\left(t_{0}+\tau^{q / 2}(\ln \tau)^{3}\right)+L \tau^{q / 2-1}(\ln \tau)^{3} \\
& \leq M\left(t_{0}+(\tau+1)^{q / 2}(\ln \tau)^{3}\right)
\end{aligned}
$$

provided that $M \geq 2 L$. This verifies $\mathcal{H}_{\tau+1}$ and completes the proof by induction.

Lemma 3.2. Fix a time $t_{0} \geq N$ and $G \in \mathcal{G}_{t_{0}}$. Then, at a later time $n \geq t_{0}$, for all $i \leq N$,

$$
\mathbf{E}\left[\operatorname{deg}_{n}^{*}\left(x_{i}^{*}\right) \mid G_{t_{0}}^{*}=G\right]=n \frac{\operatorname{deg}_{G}\left(x_{i}^{*}\right)}{t_{0}}+\tilde{O}\left(\left(n / t_{0}\right)^{5 / 6}\right)+O\left(t_{0}^{3 / 2}\right) .
$$

Proof. Notice that from Lemma 4.1, if $\tau>t_{0}$,

$$
\begin{aligned}
&\left|\mathbf{E}\left[D_{\tau}^{*}\left(S_{\tau}^{*}\right) \mid G_{t_{0}}^{*}=G\right]-\frac{2 m p}{1+p} \tau\right|>2 e^{q / 2} \tau^{5 / 6} \\
& \Rightarrow\left|D\left(S^{*}(G)\right)-\frac{2 m p}{1+p} t_{0}\right|>2 \tau^{(5-3 q) / 6} t_{0}^{q / 2} \\
& \Rightarrow 2 m t_{0}>2 \tau^{(5-3 q) / 6} t_{0}^{q / 2} \\
& \Rightarrow m^{3} t_{0}^{3 / 2}>\tau
\end{aligned}
$$

where we used $D\left(S_{G}^{*}\right) \leq 2 m t_{0}$.

Now, let $\mathcal{A}_{\tau}^{*}$ be the event

$$
\left|D_{\tau}^{*}\left(S_{\tau}^{*}\right)-\frac{2 m p}{1+p} \tau\right|<4 e \tau^{5 / 6}
$$

Then

$$
\begin{aligned}
\operatorname{Pr}\left[\neg \mathcal{A}_{\tau}^{*} \mid G_{t_{0}}^{*}=G\right] \leq & \operatorname{Pr}\left[\left|D_{\tau}^{*}\left(S_{\tau}^{*}\right)-\mathbf{E}\left[D_{\tau}^{*}\left(S_{\tau}^{*}\right) \mid G_{t_{0}}^{*}=G\right]\right| \geq 2 e \tau^{5 / 6} \mid G_{t_{0}}^{*}=G\right] \\
& +{ }^{1}\left|\mathbf{E}\left[D_{\tau}^{*}\left(S_{\tau}^{*}\right) \mid G_{t_{0}}^{*}=G\right]-\frac{2 m p}{1+p} \tau\right|>2 e \tau^{5 / 6} \\
\leq & 2 e^{-p(\ln \tau)^{2} / 8 m^{3}}+1_{m^{3} t_{0}^{3 / 2}>\tau}
\end{aligned}
$$

where the last line follows from Lemma 4.2 and Equation (3.6). 
If $\tau \geq N$, then

$$
\mathbf{E}\left[\operatorname{deg}_{\tau+1}^{*}\left(x_{i}^{*}\right) \mid G_{\tau}^{*}, G_{t_{0}}^{*}=G\right]=\operatorname{deg}_{\tau}^{*}\left(x_{i}^{*}\right)+m q \frac{\operatorname{deg}_{\tau}^{*}\left(x_{i}^{*}\right)}{2 m \tau}+m p \frac{\operatorname{deg}_{\tau}^{*}\left(x_{i}^{*}\right)}{D_{\tau}^{*}\left(S_{\tau}^{*}\right)} .
$$

Taking expectations with respect to $G_{\tau}^{*}$, in the conditional space $G_{t_{0}}^{*}=G$, we get for every $\tau \geq t_{0}$

$$
\begin{aligned}
\mathbf{E}\left[\operatorname{deg}_{\tau+1}^{*}\left(x_{i}^{*}\right) \mid G_{t_{0}}^{*}=G\right]= & \mathbf{E}\left[\operatorname{deg}_{\tau}^{*}\left(x_{i}^{*}\right) \mid G_{t_{0}}^{*}=G\right]\left(1+\frac{q}{2 \tau}\right) \\
& +m p \mathbf{E}\left[\frac{\operatorname{deg}_{\tau}^{*}\left(x_{i}^{*}\right)}{D_{\tau}^{*}\left(S_{\tau}^{*}\right)} \mid G_{t_{0}}^{*}=G\right] .
\end{aligned}
$$

But,

$$
\begin{aligned}
& \mathbf{E}\left[\frac{\operatorname{deg}_{\tau}^{*}\left(x_{i}^{*}\right)}{D_{\tau}^{*}\left(S_{\tau}^{*}\right)} \mid G_{t_{0}}^{*}=G\right]=\mathbf{E}\left[\frac{\operatorname{deg}_{\tau}^{*}\left(x_{i}^{*}\right)}{D_{\tau}^{*}\left(S_{\tau}^{*}\right)} \mid \mathcal{A}_{\tau}^{*}, G_{t_{0}}^{*}=G\right] \operatorname{Pr}\left(\mathcal{A}_{\tau}^{*} \mid G_{t_{0}}^{*}=G\right) \\
& +\mathbf{E}\left[\frac{\operatorname{deg}_{\tau}^{*}\left(x_{i}^{*}\right)}{D_{\tau}^{*}\left(S_{\tau}^{*}\right)} \mid \neg \mathcal{A}_{\tau}^{*}, G_{t_{0}}^{*}=G\right] \operatorname{Pr}\left(\neg \mathcal{A}_{\tau}^{*} \mid G_{t_{0}}^{*}=G\right) \\
& =\mathbf{E}\left[\operatorname{deg}_{\tau}^{*}\left(x_{i}^{*}\right) \mid \mathcal{A}_{\tau}^{*}, G_{t_{0}}^{*}=G\right]\left(\frac{1+p}{2 m p \tau}+\tilde{O}\left(\tau^{-7 / 6}\right)\right) \\
& \times \operatorname{Pr}\left(\mathcal{A}_{\tau}^{*} \mid G_{t_{0}}^{*}=G\right) \\
& +O\left(\operatorname{Pr}\left(\neg \mathcal{A}_{\tau}^{*} \mid G_{t_{0}}^{*}=G\right)\right) \\
& =\mathbf{E}\left[\operatorname{deg}_{\tau}^{*}\left(x_{i}^{*}\right) \mid G_{t_{0}}^{*}=G\right]\left(\frac{1+p}{2 m p \tau}\right)+\tilde{O}\left(\tau^{-1 / 6}\right) \\
& +O\left(\operatorname{Pr}\left(\neg \mathcal{A}_{\tau}^{*} \mid G_{t_{0}}^{*}=G\right)\right) \\
& =\mathbf{E}\left[\operatorname{deg}_{\tau}^{*}\left(x_{i}^{*}\right) \mid G_{t_{0}}^{*}=G\right]\left(\frac{1+p}{2 m p \tau}\right)+\tilde{O}\left(\tau^{-1 / 6}\right) \\
& +O\left(1_{m^{3} t_{0}^{3 / 2}>\tau}\right),
\end{aligned}
$$

where we used the fact $\operatorname{deg}_{\tau}^{*}\left(x_{i}^{*}\right) \leq D_{\tau}^{*}\left(S_{\tau}^{*}\right) \leq 2 m \tau$ and Equation (3.7). Therefore,

$$
\begin{aligned}
\mathbf{E}\left[\operatorname{deg}_{\tau+1}^{*}\left(x_{i}^{*}\right) \mid G_{t_{0}}^{*}=G\right]= & \mathbf{E}\left[\operatorname{deg}_{\tau}^{*}\left(x_{i}^{*}\right) \mid G_{t_{0}}^{*}=G\right]\left(1+\frac{1}{\tau}\right) \\
& +\tilde{O}\left(\tau^{-1 / 6}\right)+O\left(1_{m^{3} t_{0}^{3 / 2}>\tau}\right),
\end{aligned}
$$

and by induction, for every $n>t_{0}$,

$$
\mathbf{E}\left[\operatorname{deg}_{n}^{*}\left(x_{i}^{*}\right) \mid G_{t_{0}}^{*}=G\right]=n \frac{\operatorname{deg}_{G}\left(x_{i}^{*}\right)}{t_{0}}+\tilde{O}\left(\left(n / t_{0}\right)^{5 / 6}\right)+O\left(t_{0}^{3 / 2}\right) .
$$


Now we prove the following.

Lemma 3.3. There exists $D \geq 0$ such that the sequence

$$
\frac{\operatorname{deg}_{t}\left(s_{i}\right)}{t}-\frac{D}{t^{1 / 6}}
$$

$t \geq N$, is a sub-martingale.

Proof. Proceeding as in Lemma 3.2, let $\mathcal{A}_{\tau}$ be the event

$$
\left|D_{\tau}\left(S_{\tau}\right)-\frac{2 m p}{1+p} \tau\right|<4 e \tau^{5 / 6} .
$$

Then, if $\tau \geq m^{3} t_{0}^{3 / 2}$,

$$
\mathbf{E}\left[\operatorname{deg}_{\tau+1}\left(s_{i}\right) \mid G_{\tau}\right] \geq \operatorname{deg}_{\tau}\left(s_{i}\right)+m q \frac{\operatorname{deg}_{\tau}\left(s_{i}\right)}{2 m \tau}+m p \frac{\operatorname{deg}_{\tau}\left(s_{i}\right)}{D_{\tau}\left(S_{\tau}\right)} .
$$

Taking expectations with respect to $G_{\tau}$ we obtain

$$
\mathbf{E}\left[\operatorname{deg}_{\tau+1}\left(s_{i}\right)\right] \geq \mathbf{E}\left[\operatorname{deg}_{\tau}\left(s_{i}\right)\right]\left(1+\frac{q}{2 \tau}\right)+m p \mathbf{E}\left[\frac{\operatorname{deg}_{\tau}\left(s_{i}\right)}{D_{\tau}\left(S_{\tau}\right)}\right] .
$$

But,

$$
\begin{aligned}
\mathbf{E}\left[\frac{\operatorname{deg}_{\tau}\left(s_{i}\right)}{D_{\tau}\left(S_{\tau}\right)}\right] & \geq \mathbf{E}\left[\frac{\operatorname{deg}_{\tau}\left(s_{i}\right)}{D_{\tau}\left(S_{\tau}\right)} \mid \mathcal{A}_{\tau}\right] \operatorname{Pr}\left[\mathcal{A}_{\tau}\right] \\
& \geq \frac{1+p}{2 m p \tau}\left(1-\frac{2 e(1+p)}{m p \tau^{1 / 6}}\right) \mathbf{E}\left[\operatorname{deg}_{\tau}\left(s_{i}\right) \mid \mathcal{A}_{\tau}\right] \operatorname{Pr}\left(\mathcal{A}_{\tau}\right) \\
& \geq \frac{1+p}{2 m p \tau}\left(1-\frac{2 e(1+p)}{m p \tau^{1 / 6}}\right)\left(\mathbf{E}\left[\operatorname{deg}_{\tau}\left(s_{i}\right)\right]-2 m \tau \operatorname{Pr}\left(\neg \mathcal{A}_{\tau}\right)\right) \\
& \geq \mathbf{E}\left[\operatorname{deg}_{\tau}\left(s_{i}\right)\right]\left(\frac{1+p}{2 m p \tau}\right)-\frac{2 e(1+p)^{2}}{m p^{2} \tau^{1 / 6}}-\frac{2}{p} e^{-\frac{p(\ln \tau)^{2}}{8 m^{3}}}
\end{aligned}
$$

where we used $\operatorname{deg}_{\tau}\left(s_{i}\right) \leq D_{\tau}\left(S_{\tau}\right) \leq 2 m \tau$ and Lemma 4.2 together with Lemma 2.1.

Substituting into Equation (3.9) we see that there is a constant $D^{\prime}=D^{\prime}(m, p) \geq$ 0 such that for every $\tau \geq 1$,

$$
\mathbf{E}\left[\frac{\operatorname{deg}_{\tau+1}\left(s_{i}\right)}{\tau+1}\right] \geq \mathbf{E}\left[\frac{\operatorname{deg}_{\tau}\left(s_{i}\right)}{\tau}\right]-\frac{D^{\prime}}{\tau^{7 / 6}} .
$$

(We may have to adjust the value of $D^{\prime}$ to account for small $\tau<m^{3} t_{0}^{3 / 2}$.) 
This implies that

$$
\mathbf{E}\left[\frac{\operatorname{deg}_{\tau+1}\left(s_{i}\right)}{\tau+1}\right]-\frac{D^{\prime}}{12(\tau+1)^{1 / 6}} \geq \mathbf{E}\left[\frac{\operatorname{deg}_{\tau}\left(s_{i}\right)}{\tau}\right]-\frac{D^{\prime}}{12 \tau^{1 / 6}} .
$$

\section{The Celebrity List Gets Fixed}

We first show that the total degree of celebrities, $D_{t}^{*}\left(S^{*}\right)$, is concentrated whp and is, in expectation, a constant fraction of all edges ever added to the graph, as evident from simulation results shown in Figure 2. Then we show that $z_{t}^{*}$ and $Z_{t}^{*}$ are also concentrated whp. These results lead us to bounds on the probability of having a small gap between celebrities and non-celebrities.

Lemma 4.I. Fix $t_{0} \geq N$ and $G \in \mathcal{G}_{t_{0}}$. Let $t \geq t_{0}$, then

$$
\left|\mathbf{E}\left[D_{t}^{*}\left(S^{*}\right) \mid G_{t_{0}}=G\right]-\frac{2 m p}{1+p} t\right| \leq\left|D\left(S^{*}(G)\right)-\frac{2 m p}{1+p} t_{0}\right|\left(\frac{t e}{t_{0}}\right)^{q / 2} .
$$

Proof. Let $\tau \geq N$, then

$$
\begin{aligned}
\mathbf{E}\left[D_{\tau+1}^{*}\left(S^{*}\right) \mid D_{\tau}^{*}\left(S^{*}\right)\right] & =D_{\tau}^{*}\left(S^{*}\right)+m p+q m \frac{D_{\tau}^{*}\left(S^{*}\right)}{2 m \tau} \\
& =m p+D_{\tau}^{*}\left(S^{*}\right)\left(1+\frac{q}{2 \tau}\right) .
\end{aligned}
$$

Thus,

$$
\left|\mathbf{E}\left[D_{\tau+1}^{*}\left(S^{*}\right) \mid D_{\tau}^{*}\left(S^{*}\right)\right]-\frac{2 m p}{1+p}(\tau+1)\right|=\left|D_{\tau}^{*}\left(S^{*}\right)-\frac{2 m p}{1+p} \tau\right|\left(1+\frac{q}{2 \tau}\right) .
$$

It follows that

$$
\left|\mathbf{E}\left[D_{t}^{*}\left(S^{*}\right) \mid G_{t_{0}}=G\right]-\frac{2 m p}{1+p} t\right| \leq\left|D\left(S^{*}(G)\right)-\frac{2 m p}{1+p} t_{0}\right| \exp \left\{\sum_{\tau=t_{0}}^{t-1} \frac{q}{2 \tau}\right\},
$$

and we observe that $\sum_{\tau=t_{0}}^{t-1} 1 / \tau \leq 1+\ln \left(t / t_{0}\right)$.

Lemma 4.2. Fix $t_{0} \geq N$ and $G \in \mathcal{G}_{t_{0}}$. Let $\mathcal{B}$ denote the event $G_{t_{0}}^{*}=G$. For $t \geq t_{0}$ and $\lambda>0$,

$$
\operatorname{Pr}\left[\left|D_{t}^{*}\left(S^{*}\right)-\mathbf{E}\left[D_{t}^{*}\left(S^{*}\right) \mid \mathcal{B}\right]\right| \geq \lambda t^{1 / 2} \ln t \mid \mathcal{B}\right] \leq 2 e^{-\lambda^{2} p(\ln t)^{2} / 8 m^{3}} .
$$


Proof. We condition on $\mathcal{B}$ and omit this explicit conditioning in our expressions. Enumerate the edges $e_{1}, e_{2}, \ldots, e_{m t}$ in the order they appear. For $i>t_{0} m$, let $Y_{i}$ be the 0,1 random variable taking value 1 if and only if $e_{i}$ is incident to $S^{*}$. Then,

$$
\begin{aligned}
D_{t}^{*}\left(S^{*}\right) & =D\left(S^{*}\right)+\sum_{i=m t_{0}+1}^{m t} Y_{i}, \\
\operatorname{Pr}\left[Y_{i}=0 \mid D_{\lfloor i / m\rfloor}^{*}\left(S^{*}\right)\right] & =q\left(1-\frac{D_{\lfloor i / m\rfloor}^{*}\left(S^{*}\right)}{2 m\lfloor i / m\rfloor}\right) .
\end{aligned}
$$

We apply Azuma's inequality [Alon and Spencer 00] to show the concentration of $D_{t}^{*}\left(S^{*}\right)$. Given $t_{0} m<i \leq t m$, fix $y_{1}, \ldots, y_{i-1} \in\{0,1\}$ and let

$$
\begin{aligned}
\Delta_{\tau}(i)= & \mathbf{E}\left[D_{\tau}^{*}\left(S^{*}\right) \mid Y_{1}=y_{1}, \ldots, Y_{i-1}=y_{i-1}, Y_{i}=0\right] \\
& -\mathbf{E}\left[D_{\tau}^{*}\left(S^{*}\right) \mid Y_{1}=y_{1}, \ldots, Y_{i-1}=y_{i-1}, Y_{i}=1\right],
\end{aligned}
$$

for $\tau=\lceil i / m\rceil, \ldots, t$.

Notice that

$$
\Delta_{\tau+1}(i)=\Delta_{\tau}(i)+q \frac{m \Delta_{\tau}(i)}{2 m \tau} \text { and } \Delta_{\lceil i / m\rceil}(i) \leq m
$$

So

$$
\begin{aligned}
\Delta_{\tau}(i) & \leq m \prod_{j=\lceil i / m\rceil}^{\tau-1}\left(1+\frac{q}{2 j}\right) \\
& \leq 2 m\left(\frac{m \tau}{i}\right)^{q / 2} .
\end{aligned}
$$

Clearly $\Delta_{\tau}(I) \geq 0$; therefore,

$$
\sum_{i=t_{0} m+1}^{m t} \Delta_{t}(i)^{2} \leq 4 m^{2} \sum_{i=t_{0} m+1}^{m t}\left(\frac{m t}{i}\right)^{q} \leq 4 m^{2}(m t)^{q} \int_{m t_{0}}^{m t} x^{-q} d x \leq 4 m^{3} t / p
$$

and the lemma follows. 
Lemma 4.3. If $i \leq N$ and $0<A \ll t$, then

$$
\operatorname{Pr}\left[\operatorname{deg}_{t}^{*}\left(x_{i}^{*}\right)<A\right] \leq C\left(\frac{A}{t}\right)^{m},
$$

where $C:=C(p, m, N)$ is a constant.

Proof. We couple our graph process with an urn process: We start the process at time $t=N$ with $r=\operatorname{deg}_{N}^{*}\left(x_{i}^{*}\right)$ red balls and $b=2 N m-r$ blue balls. Each time we add an edge to the graph that is incident to $S^{*}$, we add a ball to the urn. If the edge is incident to $x_{i}^{*}$, the ball is red, otherwise it is blue. Then $R_{t}$, the number of red balls in the urn by time $t$, is equal to $\operatorname{deg}_{t}^{*}\left(x_{i}^{*}\right)$, while the total number of balls in the urns is $D_{t}^{*}\left(S^{*}\right)$.

Note that preferential attachment is equivalent to choosing an edge $e$ at random and then choosing a random end point from $e$. Therefore, this urn process follows a Polya urn process: in time $t$ given that we add a ball, the probability of adding a red ball is $R_{t} / T_{t}$, where $T_{t}$ is the total number of balls in the urns. We imagine our urn process isolated from the graph process and call adding a ball "a step" of the process. We use $s=1,2, \ldots, D_{t}^{*}\left(S^{*}\right)-2 N m$ to index the steps of the urn process.

Now, for any $0 \leq k \leq s$,

$$
\begin{aligned}
\operatorname{Pr}\left[R_{s}=\right. & r+k] \\
& =\left(\begin{array}{l}
s \\
k
\end{array}\right) \frac{r(r+1) \cdots(r+k-1) b(b+1) \cdots(b+s-k-1)}{(r+b)(r+b+1) \cdots(r+b+s-1)} \\
& =\frac{(r+b-1) !}{(s+r)(r-1) !(b-1) !} \prod_{i=1}^{r-1} \frac{k+i}{s+i} \prod_{i=1}^{r+k}\left(1-\frac{b-1}{b+s-k+i-1}\right) \\
& \leq \frac{(r+b-1) !}{(s+r)(r-1) !(b-1) !}\left(\frac{k+r-1}{s+r-1}\right)^{r-1}\left(1-\frac{b-1}{b+s+r-1}\right)^{r+k} .
\end{aligned}
$$

Therefore, if $A>0$,

$$
\begin{aligned}
\operatorname{Pr}\left[R_{s} \leq A\right] & \leq \frac{(r+b-1) !}{(s+r)(r-1) !(b-1) !} \sum_{k=0}^{A-r}\left(\frac{k+r-1}{s+r-1}\right)^{r-1} \\
& \leq \frac{(r+b-1) !}{(r-1) !(b-1) !} \int_{0}^{A / s} x^{r-1} d x \\
& \leq \frac{2^{r+b} A^{r}}{r s^{r}}
\end{aligned}
$$


Recalling that $r \geq m$ and $r+b=2 N m$ and $\operatorname{deg}_{t}^{*}\left(x_{i}^{*}\right)=R_{D_{t}^{*}\left(S^{*}\right)-2 N m}$, we get, using Lemma 4.2 with $t_{0}=N$,

$$
\begin{aligned}
\operatorname{Pr}\left[\operatorname{deg}_{t}^{*}\left(x_{i}^{*}\right) \leq A\right] \leq & \operatorname{Pr}\left[\operatorname{deg}_{t}^{*}\left(x_{i}^{*}\right) \leq A \mid D_{t}^{*}\left(S^{*}\right) \geq \frac{2 p m}{1+p} t-t^{1 / 2} \ln t\right] \\
& +\operatorname{Pr}\left[D_{t}^{*}\left(S^{*}\right)<\frac{2 p m}{1+p} t-t^{1 / 2} \ln t\right] \\
\leq & \operatorname{Pr}\left[R_{s} \leq A \mid s \geq \frac{2 p m}{1+p} t-t^{1 / 2} \ln t-2 N m\right]+e^{-p(\ln t)^{2} / 8 m^{3}} \\
\leq & \frac{2^{2 N m} A^{r}}{r\left(\frac{2 p m}{1+p} t-t^{1 / 2} \ln t-2 N m\right)^{r}}+e^{-p(\ln t)^{2} / 8 m^{3}} \\
\leq & C\left(\frac{A}{t}\right)^{r} \leq C\left(\frac{A}{t}\right)^{m}
\end{aligned}
$$

Lemma 4.4. Let $s>N$ and let $t \geq s$. Then,

$$
\operatorname{Pr}\left[\operatorname{deg}_{t}^{*}\left(x_{s}^{*}\right) \geq(t / s)^{q / 2}(\ln t)^{3}\right] \leq \exp \left(m-\frac{(\ln t)^{2}}{4}\right) .
$$

Proof. Fix $s>N$ and let $X_{\tau}=\operatorname{deg}_{\tau}^{*}\left(x_{s}^{*}\right)$ for $\tau=s, s+1, \ldots, t$.

Then, conditional on $X_{\tau}=x$, we have

$$
X_{\tau+1}=x+\text { Binomial }\left(m, \frac{q x}{2 m \tau}\right),
$$

and so

$$
\begin{aligned}
\mathbf{E}\left[e^{\lambda X_{\tau+1}} \mid X_{\tau}=x\right] & =e^{\lambda x}\left(1-\frac{q x}{2 m \tau}+\frac{q x}{2 m \tau} e^{\lambda}\right)^{m} \\
& \leq e^{\lambda x} \exp \left(\frac{q x}{2 \tau}\left(e^{\lambda}-1\right)\right) \\
& \leq \exp \left(\lambda x\left(1+q \frac{(1+\lambda)}{2 \tau}\right)\right),
\end{aligned}
$$

for any $\lambda \leq 1$.

Thus,

$$
\mathbf{E}\left[e^{\lambda X_{\tau+1}}\right] \leq \mathbf{E}\left[\exp \left(X_{\tau} \lambda\left(1+\frac{q(1+\lambda)}{2 \tau}\right)\right)\right] .
$$

If we put $\lambda_{\tau-1}=\lambda_{\tau}\left(1+\frac{q\left(1+\lambda_{\tau}\right)}{2 \tau}\right)$ and take $\lambda_{t}=\lambda$ small enough such that

$$
\lambda_{\tau} \leq \Lambda=\min \left\{1, \frac{1}{\ln (t / s)}\right\} \text { for } \tau=s, \ldots, t,
$$


then Equation (4.2) implies

$$
\mathbf{E}\left[e^{\lambda_{\tau+1} X_{\tau+1}}\right] \leq \mathbf{E}\left[e^{\lambda_{\tau} X_{\tau}}\right] \quad \text { for } \tau=s+1, \ldots, t-1 .
$$

Hence,

$$
\mathbf{E}\left[e^{\lambda X_{t}}\right]=\mathbf{E}\left[e^{\lambda_{t} X_{t}}\right] \leq \mathbf{E}\left[e^{\lambda_{s} X_{s}}\right]=e^{m \lambda_{s}} .
$$

We can write

$$
\lambda_{\tau-1} \leq \lambda_{\tau}\left(1+\frac{(1+\Lambda) q}{2 \tau}\right)
$$

then

$$
\begin{aligned}
\lambda_{s} & \leq \lambda \prod_{\tau=s}^{t}\left(1+\frac{(1+\Lambda) q}{2 \tau}\right) \\
& \leq 2 \lambda(t / s)^{(1+\Lambda) q / 2}
\end{aligned}
$$

(the 2 bounds $e^{\gamma+1 / 2 t}$ where $\gamma$ is Euler's constant)

$$
\leq 2 e^{q / 2} \lambda(t / s)^{q / 2}
$$

and therefore we can take $\lambda=\frac{\Lambda}{2 e^{q / 2}}(s / t)^{q / 2}$ and get Equation (4.3).

Putting $u=(t / s)^{q / 2}(\ln t)^{3}$, we get

$$
\begin{aligned}
\operatorname{Pr}\left(X_{t} \geq u\right) & \leq e^{m \lambda_{s}-\lambda u} \\
& \leq \exp \left(\Lambda m-\frac{\Lambda(\ln t)^{3}}{4}\right) \\
& \leq \exp \left(m-\frac{(\ln t)^{2}}{4}\right)
\end{aligned}
$$

Lemma 4.5. If $0<A \ll t$, then

$$
\operatorname{Pr}\left[z_{t}-Z_{t} \leq A\right] \leq C\left(\frac{A}{t}\right)^{m},
$$

where $C:=C(p, m, N)$ is a constant.

Proof. Let $C^{\prime}$ be the constant from Lemma 4.3. Then,

$$
\operatorname{Pr}\left[z_{t}^{*}<2 A\right] \leq C^{\prime}\left(\frac{2 A}{t}\right)^{m} .
$$

Also, from Lemma 4.4,

$$
\operatorname{Pr}\left[Z_{t}^{*} \geq t^{q / 2}(\ln t)^{3}\right] \leq t \exp \left(m-\frac{(\ln t)^{2}}{4}\right) .
$$


Using Lemma 2.1, and putting Equations (4.4) and (4.5) together, we get that if $A \geq t^{1 / 2}$ and $t$ is sufficiently large,

$$
\begin{aligned}
\operatorname{Pr}\left[z_{t}-Z_{t} \leq A\right] & \leq \operatorname{Pr}\left[z_{t}^{*}-Z_{t}^{*} \leq A\right] \\
& \leq \operatorname{Pr}\left[z_{t}^{*}<2 A\right]+\operatorname{Pr}\left[Z_{t}^{*} \geq A\right] \\
& \leq C^{\prime}\left(\frac{2 A}{t}\right)^{m}+t \exp \left(m-\frac{(\ln t)^{2}}{4}\right) \\
& \leq 4^{m} C^{\prime}\left(\frac{A}{t}\right)^{m} .
\end{aligned}
$$

\section{Proof of Theorem 1.2}

Proof. Fix $i \leq N$. It follows from the (sub-)martingale convergence theorem (see [Durrett 91]) and Lemma 3.3 that

$$
L=\lim _{t \rightarrow \infty} \frac{\mathbf{E}\left[\operatorname{deg}_{t}\left(s_{i}\right)\right]}{t} \text { exists. }
$$

We have to show that $L$ is strictly positive and bounded away from zero. But, $L \geq m / N$ follows immediately from Lemmas 2.1 and 3.2. This proves the first part of Theorem 1.2.

Our proof of the second part of Theorem 1.2 is a little more complicated, due to the fact that we want to estimate $\bar{d}_{k}(n) / n$ reasonably accurately (as opposed to using martinagale convergence as we did in Part (a)). Let $\gamma_{t}=Z_{t}-z_{t}$, and let $t_{0}$ be the first time that $\gamma_{t} \geq n^{q / 2}$. Let $t_{1}>t_{0}$ be the first time after $t_{0}$ such that $\gamma_{t} \leq m$.

Notice that in the time interval $\left[t_{0}, t_{1}\right], S_{t}$ is fixed, therefore, conditional on $G_{t_{0}}=G_{t_{0}}^{*}$, processes $\mathcal{P}$ and $\mathcal{P}^{*}$ coincide for every $t$ such that $t_{0} \leq t \leq t_{1}$.

Thus,

$$
\begin{aligned}
\mathbf{E}\left[d_{k}\left(G_{n}\right)\right]= & \mathbf{E}\left[d_{k}\left(G_{n}\right) \mid t_{0}>n\right] \operatorname{Pr}\left[t_{0}>n\right]+\mathbf{E}\left[d_{k}\left(G_{n}\right) \mid t_{1} \leq n\right] \operatorname{Pr}\left[t_{1} \leq n\right] \\
& +\sum_{t=1}^{n} \sum_{G \in \mathcal{G}_{t}} \mathbf{E}\left[d_{k}^{*}(n) \mid G_{t_{0}}=G\right] \operatorname{Pr}\left[t_{0}=t, G_{t}=G\right] \\
= & O(n)\left(\operatorname{Pr}\left[t_{0}>n\right]+\operatorname{Pr}\left[t_{1} \leq n\right]\right) \\
& +\sum_{t=1}^{n} \sum_{G \in \mathcal{G}_{t}}\left(\frac{2 n}{2+m q} \prod_{i=m+1}^{k} \frac{i-1}{i+2 / q}+\tilde{O}\left(t+n^{q / 2}\right)\right) \\
& \times \operatorname{Pr}\left[t_{0}=t, G_{t}=G\right]
\end{aligned}
$$




$$
\begin{aligned}
= & \frac{2 n}{2+m q} \prod_{i=m+1}^{k} \frac{i-1}{i+2 / q} \\
& +O(n)\left(\operatorname{Pr}\left[t_{0}>n\right]+\operatorname{Pr}\left[t_{1} \leq n\right]\right)+\tilde{O}\left(n^{q / 2}\right)+\tilde{O}(1) \mathbf{E}\left[t_{0} \mid t_{0} \leq n\right] .
\end{aligned}
$$

It only remains to show that the contributions from Equation (5.1) are $\tilde{O}\left(n^{q / 2}\right)$. Let $C$ be the constant defined in Lemma 4.5

Firstly,

$$
\operatorname{Pr}\left[t_{0}>n\right] \leq \operatorname{Pr}\left[\gamma_{n} \leq n^{q / 2}\right] \leq C\left(\frac{n^{q / 2}}{n}\right)^{m}=o\left(n^{-1}\right)
$$

and as $t_{0} \geq n^{q / 2} / m$

$$
\operatorname{Pr}\left[t_{1} \leq n\right] \leq \sum_{t=n^{q / 2} / m}^{n} \operatorname{Pr}\left[\gamma_{t} \leq m\right] \leq \sum_{t=n^{q / 2} / m}^{n} C\left(\frac{m}{t}\right)^{m}=O\left(n^{-m q / 2}\right)=O\left(n^{-1}\right) .
$$

Finally,

$$
\mathbf{E}\left[t_{0} \mid t_{0} \leq n\right] \leq \sum_{t=N}^{n} \operatorname{Pr}\left[t_{0} \geq t\right] \leq n^{q / 2}+\sum_{t=n^{q / 2}}^{n} C\left(\frac{n^{q / 2}}{t}\right)^{m}=O\left(n^{q / 2}\right) .
$$

\section{Concluding Remarks}

We have shown that modeling the influence of a search engine within the preferential attachment framework leads to a qualitative change in the familiar powerlaw degree distribution. Each of a collection of celebrities captures a constant fraction of the total degree of the graph, and the degree of the remaining nodes follow a steeper power law.

Our model differs from reality in many obvious ways: edges are undirected, outlinks are not modified after creation, pages do not die, there is no topic-based clustering, and there is no propensity toward forming bipartite cores as in the copying model.

Despite these limitations, our results lend support to recent articles by political scientists [Hindman et al. 03] in the popular press expressing apprehension about the extent to which search engines concentrate the collective attention of web surfers to "mainstream" websites. Another study [Pandey et al. 05] involving live users rating jokes confirms the existence of the entrenchment effect and shows that it can be reduced by limited randomization of the ranked list.

However, there is no verdict yet on the severity of the entrenchment effect of search engines in practice. A recent study [Fortunato et al. 05] claims that 
the use of search engines actually has an egalitarian effect, in part owing to the diversity of query words used in searches. Enhancing entrenchment models with link-copying and query effects would be natural candidates for future work.

Acknowledgments. We would like to thank the referee for his/her persistence in suggesting the use of martingale convergence in the proof of Theorem 1.2(a). Research by the first author was supported in part by NSF grant CCR-0200945. Research by the second and third authors was done while visiting the Department of Computer Science, Carnegie Mellon University.

\section{References}

[Alon and Spencer 00] N. Alon and J. Spencer. The Probabilistic Method, Second Edition. New York: Wiley Interscience, 2000.

[Barabási and Albert 99] A. Barabási and R. Albert. "Emergence of Scaling in Random Networks." Science 286 (1999), 509-512.

[Brin and Page 98] S. Brin and L. Page. "The Anatomy of a Large-Scale Hypertextual Web Search Engine." Proceedings of WWW 107-117.

[Cho and Roy 04] J. Cho and S. Roy. "Impact of Search Engines on Page Popularity." In Proceedings of the International Conference on World Wide Web, pp. 20-29. New York: ACM Press, 2004.

[Cooper and Frieze 03] C. Cooper and A. M. Frieze. "A General Model of Undirected Web Graphs." Random Structures and Algorithms 22 (2003), 311-335.

[Drinea et al. 02] E. Drinea, A.M. Frieze, and M. Mitzenmacher. "Balls and Bins Models with Feedback." In Proceedings of the Thirteenth Annual ACM-SIAM Symposium on Discrete Algorithms, pp. 308-315. Philadelphia, PA: SIAM, 2002.

[Durrett 91] R. Durrett. Probability: Theory and Examples. Belmont, CA: Wadsworth, 1991.

[Flaxman et al. 05] A. Flaxman, A. M. Frieze, and T. I. Fenner. "High-Degree Vertices and Eigenvalues in the Preferential Attachment Graph." Internet Mathematics 2 (2005), 1-20.

[Fortunato et al. 05] S. Fortunato, A Flammini, F. Menczer, and A. Vespignani. "The Egalitarian Effect of Search Engines." Preprint available at http://arxiv.org/abs/ cs/0511005, 2005.

[Hindman et al. 03] M. Hindman, K. Tsioutsiouliklis, and J. A. Johnson. "Googlearchy: How a Few Heavily-Linked Sites Dominate Politics on the Web." Annual Meeting of the Midwest Political Science Association, 2003.

[Kleinberg et al. 99] J. Kleinberg, R. Kumar, P. Raghavan, S. Rajagopalan, A. Tomkins. "The Web as a Graph: Measurements, Models and Methods." In Computing and Combinatorics: 5th Annual International Conference, COCOON '99, Tokyo, Japan, July 26-28, 1999, Proceedings, Lecture Notes in Computer Science 1627, pp. 1-18. Berlin: Springer, 1999. 
[Kumar et al. 00] R. Kumar, P. Raghavan, S. Rajagopalan, D. Sivakumar, A. Tomkins, and E. Upfal. "Stochastic Models for the Web-Graph." In Proceedings of the 41st Annual Symposium on Foundations of Computer Science, pp. 57-65. Los Alamitos, CA: IEEE Computer Society, 2000.

[Pandey et al. 05] S. Pandey, S. Roy, C. Olston, J. Cho, and S. Chakrabarti. "Shuffling a Stacked Deck: The Case for Partially Randomized Ranking of Search Engine Results." In Proceedings of the 31st International Conference on Very Large Data Bases, pp. 781-792. New York: ACM Press, 2005.

[Pennock et al. 02] D. M. Pennock, G. W. Flake, S. Lawrence, C. Lee Giles, and E. J. Glover. "Winners Don't Take All: Characterizing the Competition for Links on the Web." Proceedings of the National Academy of Sciences 99:8 (2002), 5207-5211.

[Johnson and Kotz 77] N.L. Johnson and S. Kotz. Urn Models and Their Application: An Approach to Modern Discrete Probability Theory. New York: Wiley, 1977.

Alan Frieze, Department of Mathematical Sciences, Carnegie Mellon University, Pittsburgh, PA 15213-3890 (alan@random.math.cmu.edu)

Juan Vera, Department of Mathematical Sciences, Carnegie Mellon University, Pittsburgh, PA 15213-3890 (jvera@cc.gatech.edu)

Soumen Chakrabarti, Indian Institute of Technology, Bombay, Powai, Mumbai 40076, India (soumen@cse.iitb.ac.in)

Received January 6, 2006; accepted May 21, 2007. 